\title{
Effective Technological Equipment for Mass Production of Entomophagous Insects and Mites Used for Biological Control
}

\author{
Valentina Krutyakova \\ Engineering and Technology Institute "Biotechnics", Odessa 65067, Ukraine
}

\begin{abstract}
Mass production of entomophagous insects and mites is necessary for the practical use of biological method of plant protection in agriculture. For many insects, breeding is carried out at the level of industrial technology with the appropriate equipment. The main purpose of the research was to improve the technical and economic performance of equipment in the production of entomophagous insects and their hosts by optimizing equipment design and technocenosis management techniques. In laboratory experiments with using the planning of multifactor experiments, the parameters of cages for breeding were determined optimally. On this basis, sets of equipment for the trichogram, brakon, lacewort and phytoseulatus have been developed. The specific productivity of new equipment provides the necessary profitability of production.
\end{abstract}

Key words: Production, entomophagous insects and mites, technological equipment, cage, productivity, profitability.

\section{Introduction}

In augmentative biological control, invertebrate organisms are seasonally released in large numbers to reduce pests. Today, it is applied on more than 30 million ha worldwide. Currently, about 230 species of entomophagous insects are bred under artificial conditions and successfully used to protect plants in open and closed ground [1]. For many insects, mass breeding is carried out in very large quantities [2]. In fact, this is an industrial production, which is based on industrial technologies and appropriate equipment. However, publication on the description of such equipment is very small.

In Ukraine, since the 90 s of the last century, a trichogram was massively bred. About 300 production bio-laboratories were built, which were equipped with the necessary mechanized equipment $[3,4]$. Currently, about 20 enterprises are engaged in breeding insects for biological protection.

Corresponding author: Valentina Krutyakova, Ph.D., research field: economic science.
The Engineering and Technological Institute "Biotechnica" of the National Academy of Agrarian Sciences of Ukraine in Odessa is engaged in the development of equipment for insect breeding. At the institute, experimental design works are based on their own research on laboratory and mass breeding of insects and mites. The research is aimed at optimizing the technical and economic parameters of the equipment [5].

The main goal of the research was to increase the productivity of equipment kits in the production of entomophagous insects and their hosts by optimizing the design of equipment and methods of crop management. Productivity was estimated by specific indicators, i.e., the number of insects per cycle per unit area (volume) of the device or per unit of raw materials.

\section{Materials and Methods}

The technological process of mass breeding of entomophagous insects is carried out on specific equipment, which has built on a modular basis. The 
module is defined as a basic set of equipment in which a complete technological process takes place with a minimum capacity [5].

The necessary increase in performance is achieved by a proportional increase in the number of modules. Technological apparatuses and machines of modular kits are divided into the following main groups:

(1) To ensure a certain life stage of development of insects;

(2) For short-term manipulation with insects;

(3) For storing insects;

(4) For heat and air handling and air transportation;

(5) For the preparation and manufacture of feed substrates.

The main object of research was the first group, which is reduced to the creation in artificial conditions of a camera or another device of a particular design, which is called as a "cage" [6]. "Cage" must have clearly defined boundaries, physical or conditional boundaries, allowing it to be distinguished from other similar units. In the inner space of the cage, favorable conditions are created for the development of a living organism or one of the stages of its ontogenesis. Consequently, the cage can be called the designated volume of space, separated from other similar volumes by physical and/or conditional boundaries in which the technological cycle of breeding is realized and ends with the collection of products (final or intermediate). The device of the cage is the key factor determining the level of productivity of the cultivated species under artificial conditions.

In laboratory studies on the basis of methods for planning multifactorial experiments, optimal values of productivity and viability were found. Input factors were: insect density, cage height or substrate layer thickness, composition and amount of fodder substrate. On the basis of the data obtained, the technical requirements for the equipment are determined. The results of the development of a cage for specific entomophagous insects are given below.

\section{Results and Discussion}

After preliminary research on model, the development of equipment sets was carried out, and then their production. Production tests with insects have tested the efficiency and effectiveness of the equipment, as described below.

\subsection{A Modular Set of Trichogramma Production}

A modular set of trichogramma production equipment [7] is shown in Fig. 1. Trichogramma breeding is performed on eggs of grain moth (Sitotroga cerealella). The main equipment of the kit is the multiplier (Fig. 1a) [8]. Its design is characterized by a high bulk density of grain moth eggs, an increased percentage of parasitic eggs and the exclusion from the cycle of individuals with low flying activity.

The multiplier has two chambers into which blocks of plates (Fig. 1b) with eggs of grain moth are inserted through the sliding doors. The cameras have forced ventilation. In the chamber, there are upper and lower light sources of low intensity. The automatic control of these luminaires ensures the movement of the trichogramma along the plates, which increases the probability of parasitic eggs.

Trichogramma is introduced into the chamber in the container (canister for insects). To hit the plate with eggs, it must fly a predetermined distance, which can be adjusted. This allows us to reject substandard individuals.

The block of plates (Fig. 1b) is made of metal. Its design allows you to conduct technological operations without completely dismantling the plates. Variants of blocks (Figs. 1b and 1c) with 15-22 plates are offered, which contain from $150 \mathrm{~g}$ to $220 \mathrm{~g}$ of grain moth eggs. The application of grain moth eggs to the plates is carried out in the device (Fig. 1d).

The productivity of the multiplier per cycle is $440 \mathrm{~g}$ of eggs of a grain moth infected with a trichogramma. Dimension is $1.6 \mathrm{~m} \times 1.6 \mathrm{~m} \times 1.7 \mathrm{~m}$. 

Insects and Mites Used for Biological Control
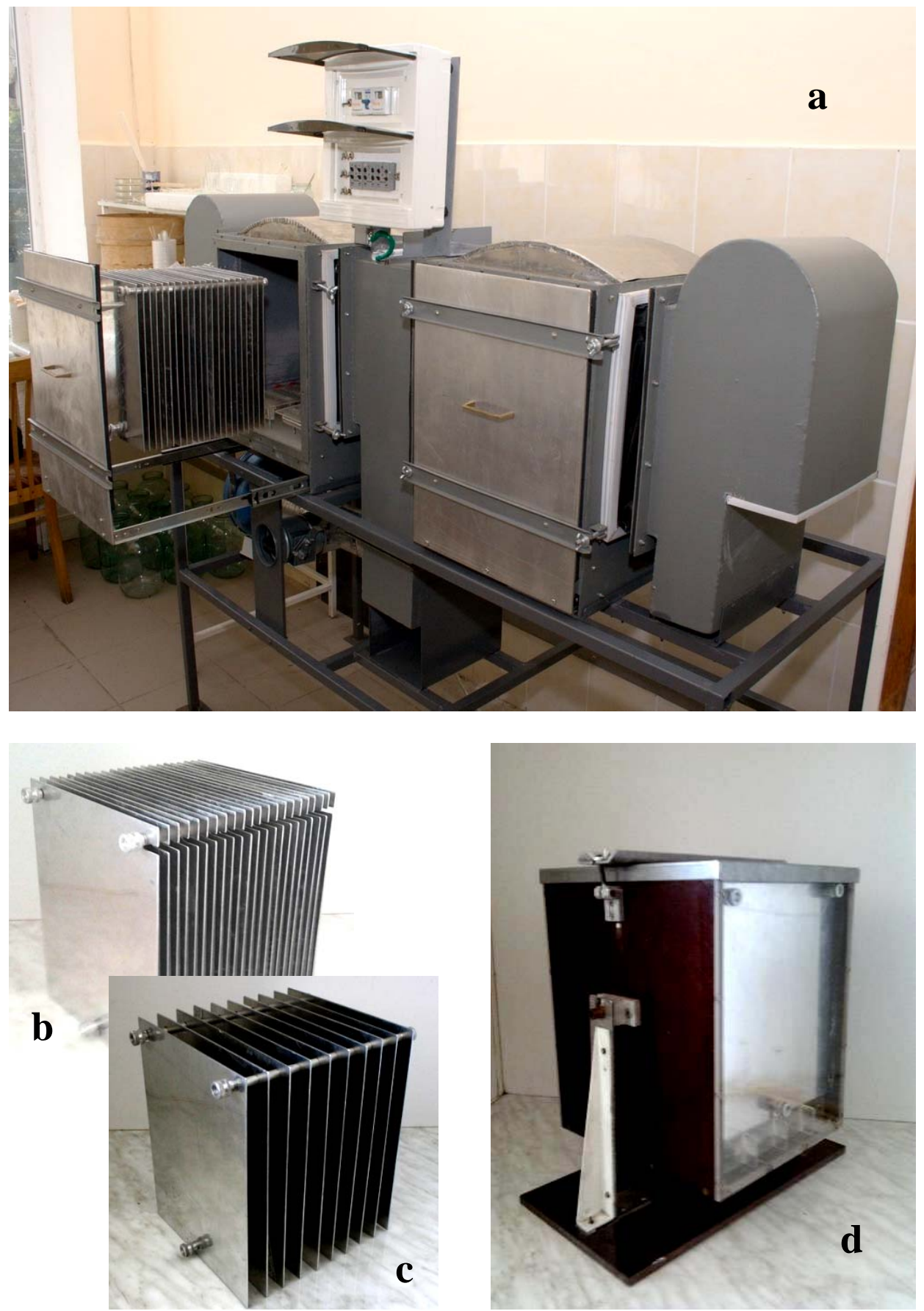

Fig. 1 The equipment for the production of the trichogramma.

\subsection{A Modular Set of Bracon Production}

A modular set of equipment for the production of a bracon (Bracon hebetor Say) [9] is shown in Fig. 2. It differs from the analogues in that the bracelet is produced in an environmentally friendly package, convenient for transportation and settlement-straw briquettes (Fig. 2f). Briquettes are a vital space for its development and at the same time a settlement device. The settling takes place in the pupa stage, which is the most resistant to negative environmental factors, unlike the usual method of settling in the adult stage.

The modular set of equipment for the production of a bracon includes: cuvettes for mill fire (230 pcs), feed 
chopper (1 pc), butterfly moth collection device (1 pc), cages for holding and collecting butterflies eggs for mills (5 pcs), pallets (5 pcs), shelving for production of briquettes ( 2 pcs), device for driving caterpillars to
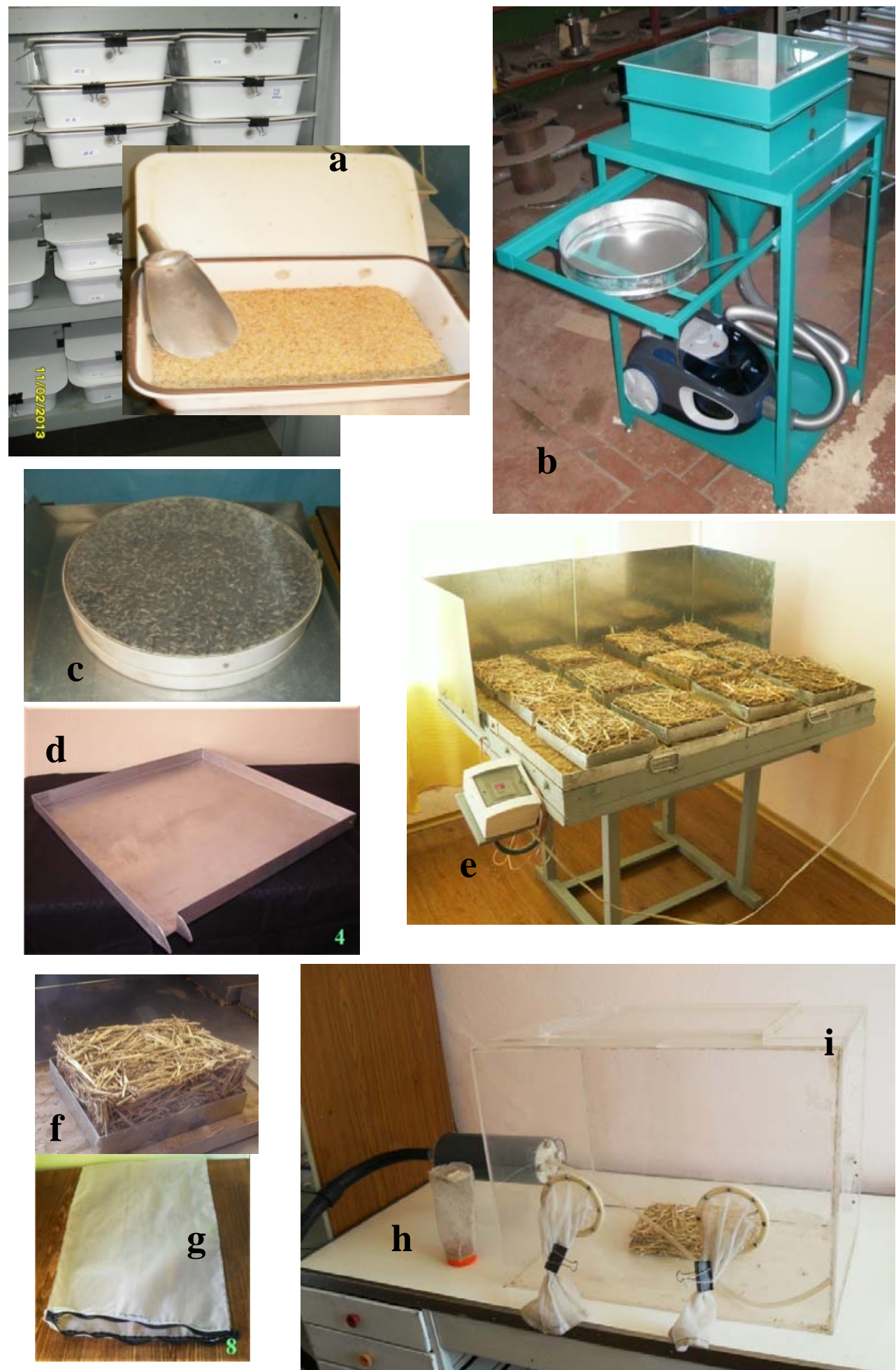

Fig. 2 The equipment for the production of the bracon. for imago of a brakon ( $40 \mathrm{pcs}$ ).
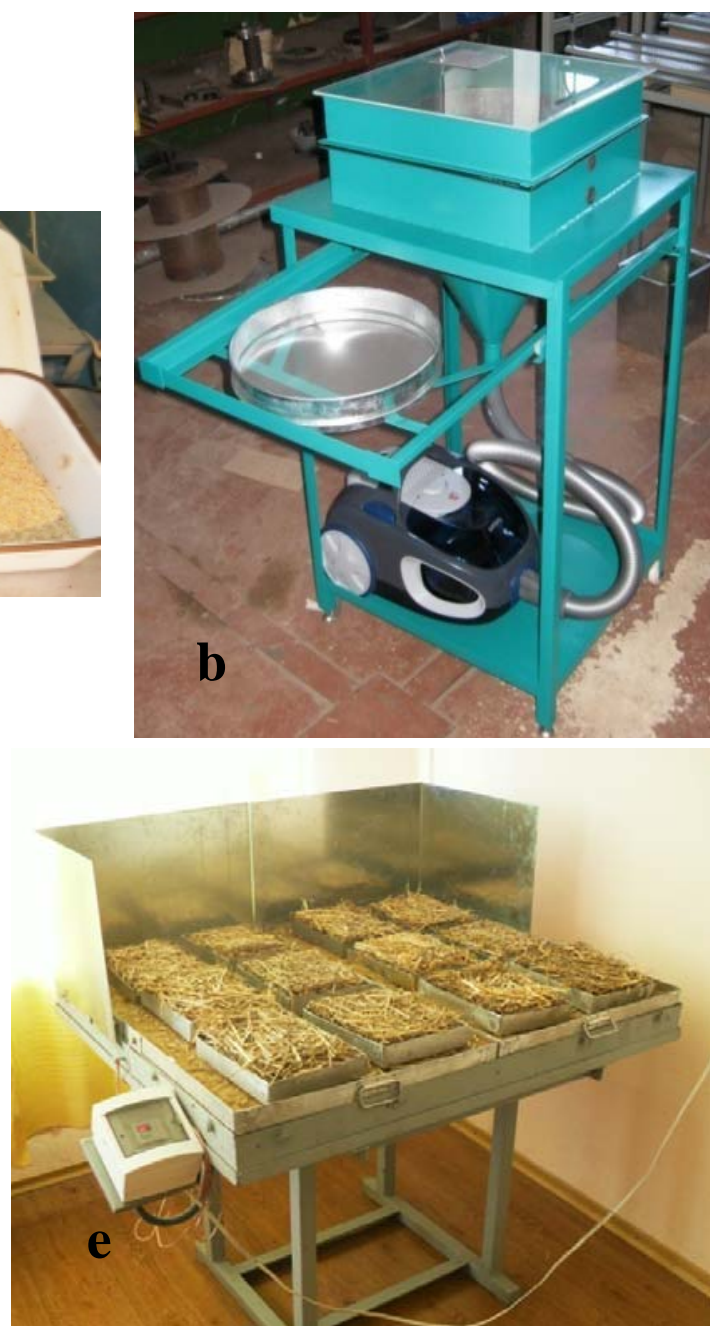

briquettes ( $1 \mathrm{pc})$, box for collection of imago of a brakona ( $1 \mathrm{pc})$, cases for briquettes (10 pcs) and cages

The larvae of the brakon are fed on the caterpillars 
of the mill fire (Ephestia kuehniella) [10]. The caterpillars are fed on a grain of barley or maize crushed in a feed mill. The millstone develops to caterpillars or butterflies in plastic cuvettes (Fig. 2a) with a cover of $470 \mathrm{~mm} \times 340 \mathrm{~mm} \times 100 \mathrm{~mm}$, which are placed on the shelves. The collection of butterflies from cuvettes to cages is carried out with the aid of device (Fig. 2b), which operates on the principle of an exhauster with an electric drive.

Cages (Fig. 2c) for the content of imago mills have a cylindrical shape with a diameter of $395 \mathrm{~mm}$ and a height of $75 \mathrm{~mm}$. The walls of the cage are made of plastic, and the upper and lower sides of it are covered with a large mesh. Each cage is recommended to accommodate no more than 5,000 butterflies. The cages filled with butterflies are placed on a shelf, and a tray for collecting eggs (Fig. 2d) is placed under each cage.

The equipment makes it possible to receive 20-25 thousand caterpillars of a mill fire every day. The device for driving caterpillars to briquettes (Fig. 2e) is designed to distill the caterpillars of the mill fire from the nutrient medium by heating it and collecting it in briquettes from straw. In briquettes on caterpillars, a brakon develops. Briquettes shown in Fig. 2f are placed in covers in Fig. 2g, which are designed to hold the imago of the brakon during its flight.

In box (Fig. 2i), using an exhauster, an imago of a brakon is collected. Productivity is three thousand imago of a brakon per hour. Overall dimensions is $1,210 \mathrm{~mm} \times 780 \mathrm{~mm} \times 480 \mathrm{~mm}$. Cage in Fig. $2 \mathrm{~h}$ is designed to contain the adult of the brakon before the infection of the caterpillars, with the capacity of 200 adults.

Compared with known technologies, new processes have been developed for distilling caterpillar mills from the fodder substrate and for breeding and dispersing the parasite in straw briquettes.

The total capacity of the equipment set is 20 bricks per day or 20-24 thousand dolls a day. The weight of the equipment set is $980 \mathrm{~kg}$, and the area of placement is $81 \mathrm{~m}^{2}$.

\subsection{A Modular Set of Equipment of the Common Green Lacewing Production}

A modular set of equipment for the production of the common green lacewing (Chrysopa carnea) [11] is shown in Fig. 3. As a food for feeding larvae of lace-eyed larvae, waste from the production of grain moth eggs are not suitable for trichogram breeding, and butterfly moths after the collection of eggs are used. The larvae are found in group cages, in contrast to the old methods, in which the individual feeding the predator in honeycombs was used.

The set includes: racks ( 3 pcs), cages for larvae (30 pcs), cages for imago (75 pcs), incubation units for eggs (3 pcs), ventilation unit (1 pc), and replacement covers for cages with imago (150 pcs).

The cage for larvae (Fig. 3a) has dimensions of 15 $\mathrm{mm} \times 250 \mathrm{~mm} \times 250 \mathrm{~mm}$ [12]. The walls are made of plexiglas, the lid is made of metal and the bottom is made of capron mesh. From one cage, you can get 900-1,100 cocoons of the golden-eyed one. It has been proved that butterflies, which are food for the larvae, simultaneously structure the habitat, reducing the frequency of contact between individuals of the predator This allows you to feed the larvae to the cocoons for group maintenance. The developed composition of the feed mixture allowed reducing the consumption of eggs of grain moth. Survival of larvae is $65 \%-70 \%$ against $40 \%$ according to traditional technology.

Cage imago (Fig. 3b) has dimensions $30 \mathrm{~mm} \times 250$ $\mathrm{mm} \times 250 \mathrm{~mm}$ [13]. The walls are made of plexiglass, and the bottom is made of capron mesh. The metal cover on the underside is covered with a cloth, on which eggs are deposited. Inside the cage, there is a feeder and a drinking bowl. Imago golden lilies are fed dry food, consisting of powdered sugar and yeast powder. The new design of cages and feed allows a ninefold increase in the density of insects. Every day 3-4 thousand eggs or 120-150 thousand eggs are taken from the cage for $55 \mathrm{~d}$. 

Insects and Mites Used for Biological Control

The incubation unit (Fig. 3d) is attached to the wall. It is designed to accommodate lids with eggs of goldeneye, which were removed from the cages for adults. The ventilation unit (Fig. 3c) performs the function of holding the imago in the cage cavity during feeding and replacing the lid with eggs, as well as separating the cocoons from the nutrient medium.

The kit is placed in a room of $15-18 \mathrm{~m}^{2}$ and allows to get approximately 190-200 thousand larvae of the 2nd age or 200 thousand predator eggs per day. This amount is sufficient to protect $0.5-1$ ha of closed or open ground.
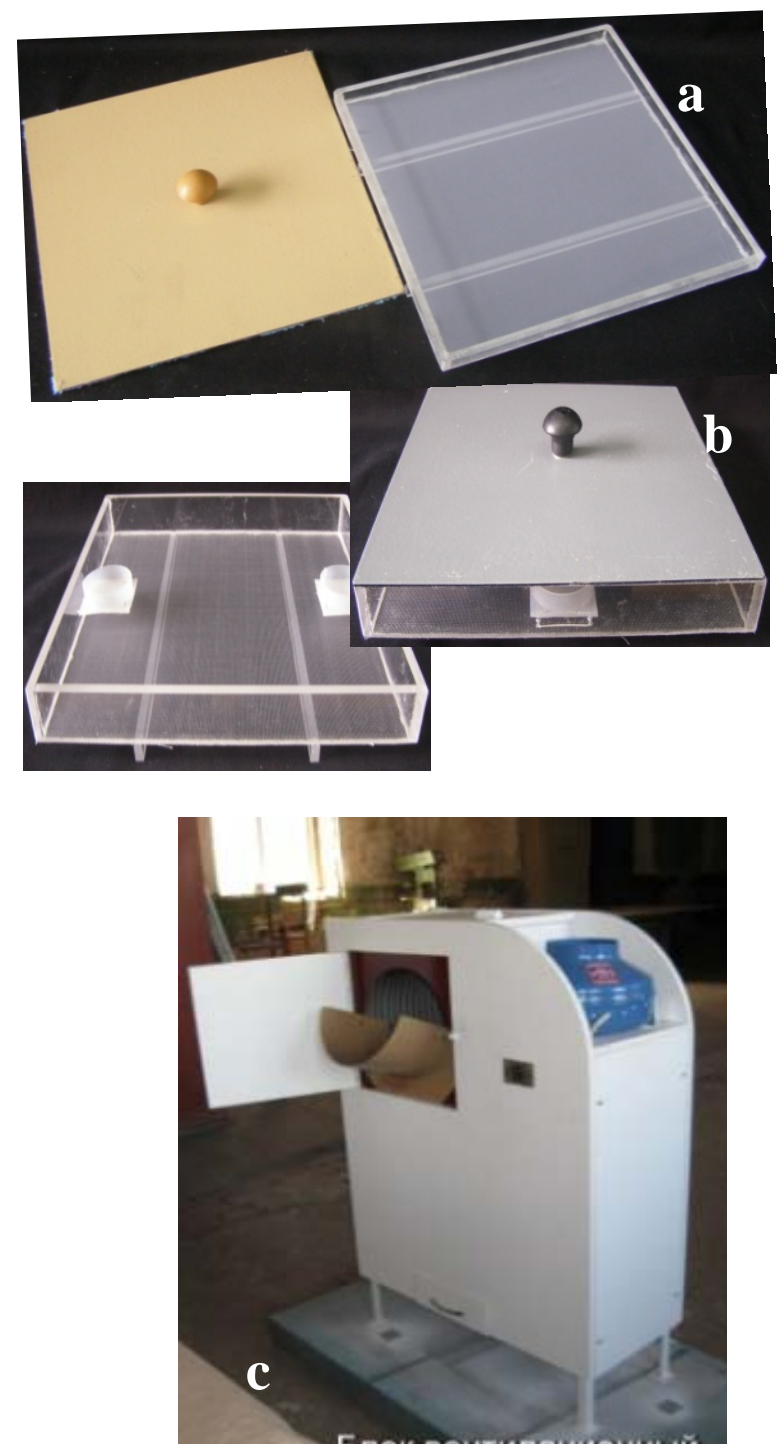

The introduction of this kit in bio-laboratories for the production of the trichogram will additionally produce 300-350 thousand eggs of lacewort per day in terms of 1 ton of dry grain.

\subsection{A Set of Equipment for Phytoseiulus Production}

A set of equipment for the mass-rearing of the phytoseiulus (Phytoseiulus persimilis) is shown in Fig. 4 [14]. As a food, a spider mite (Tetranychus urticae) is used, which is bred on soy. Their cultivation is carried out on universal shelves (Fig. 4a) with lighting with a total area of about $5.4 \mathrm{~m}^{2}$ and no features.
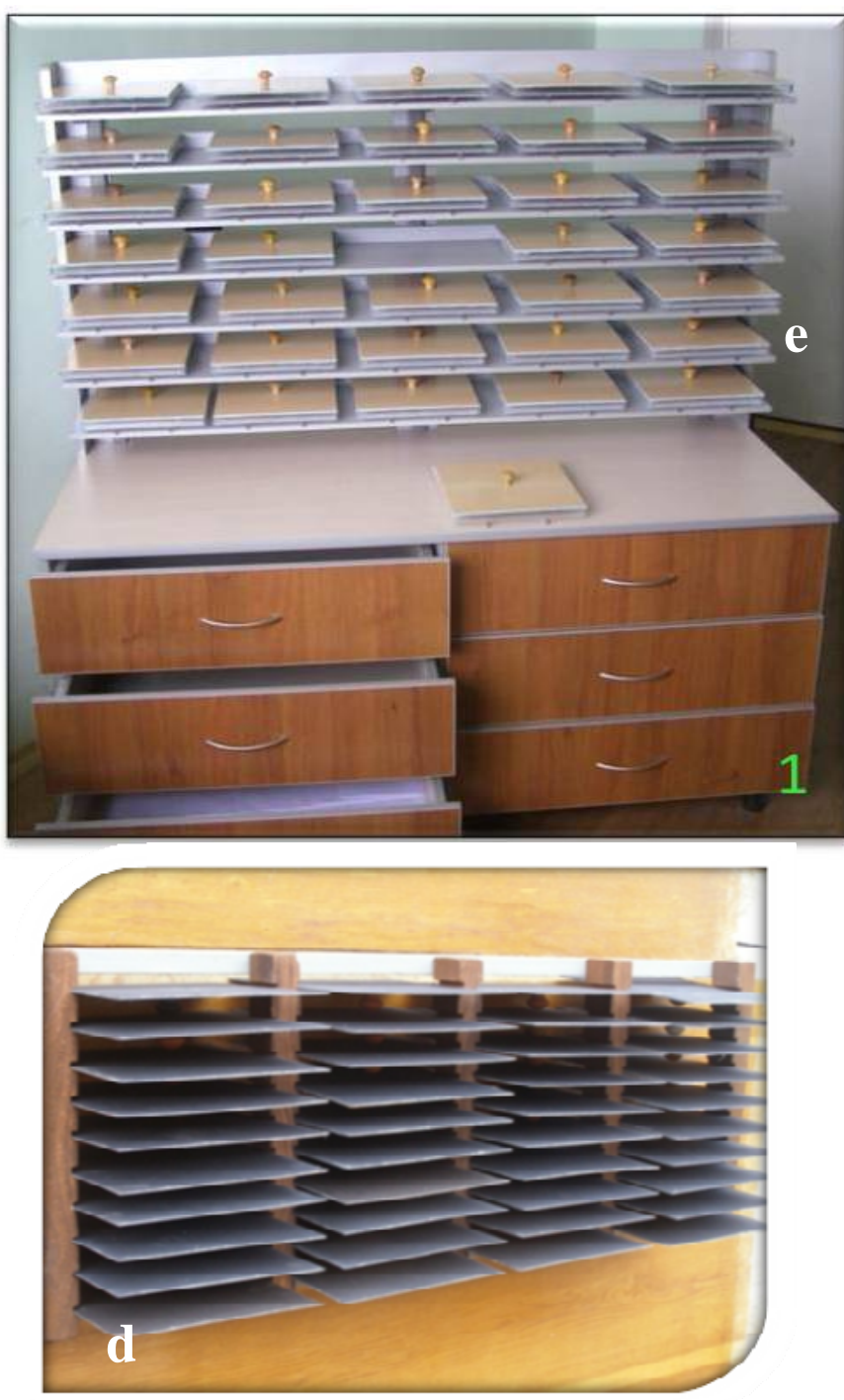

Fig. 3 The equipment for the production of the common green lacewing. 

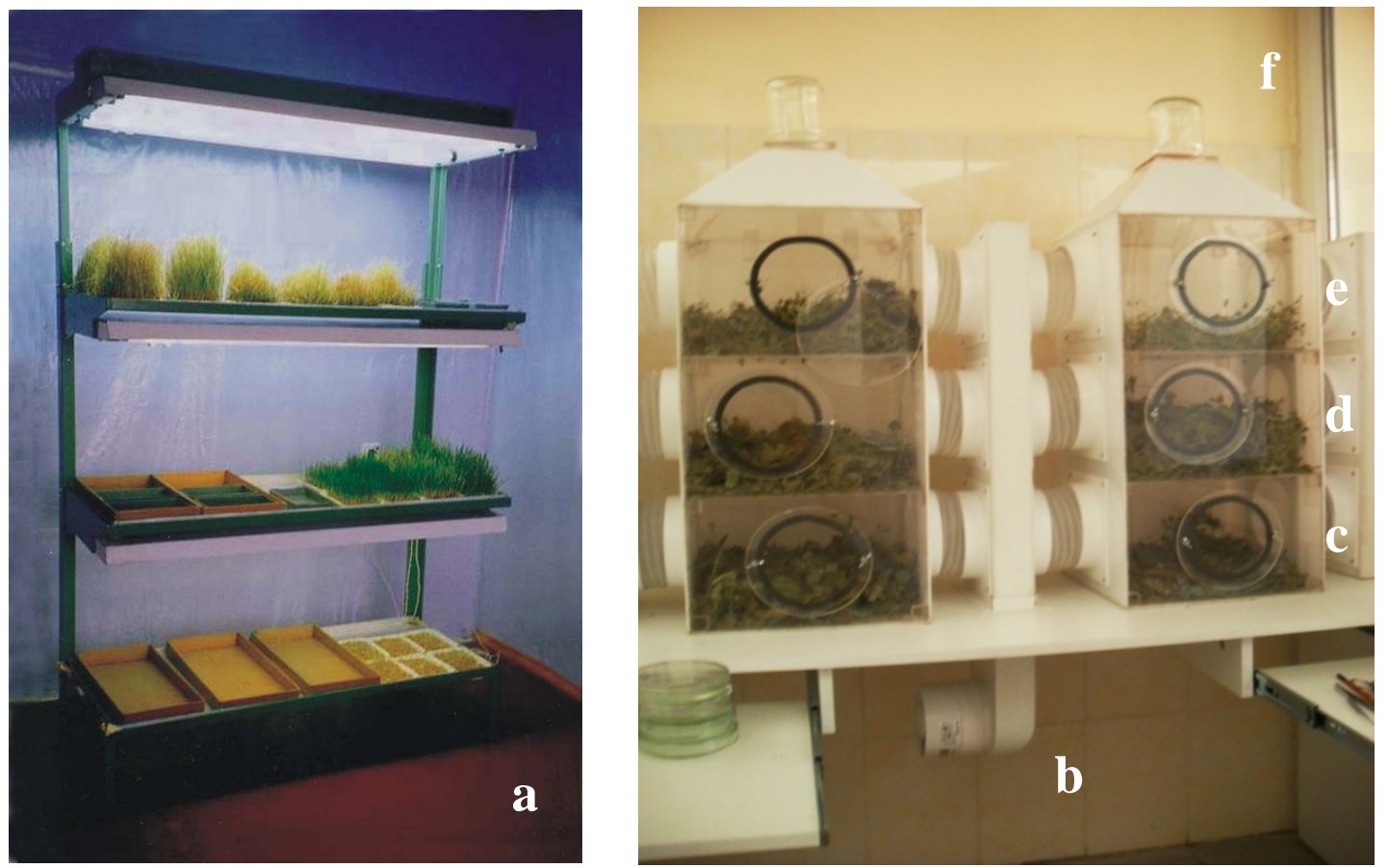

Fig. 4 The equipment for the mass-rearing of the Phytoseiulus.

Phytoseulen cultivation is carried out in module (Fig. 4b) which consists of two multi-tier cages and a ventilation system. In the cage with the help of horizontal perforated partitions, three chambers are distinguished, which have on the side walls and on the partitions manipulation hatches .In the chamber of the upper tier (Fig. 4e), plants with a spider mite are loaded through the side hatch. Then, about 3,000 phytoseiulus are introduced in the adult stage. After 2 $\mathrm{d}$, the lab technician opens the hatch in the septum through the side hatch and moves the plants with mites to the lower tier (Fig. 4d). The vacated place is again loaded with soybean plants with a spider mite.

Thus, every two days, the plants move to a lower level. When the plants reach the lowest level (Fig. $4 \mathrm{c}$ ), they stand for $2 \mathrm{~d}$ and then were removed. The mobile phases of the predatory tick are moved to the upper levels of the boxes in search of a victim. To collect the predator, stop feeding for 2-3 d. With a decrease in the amount of food, the phytosialus leaves the plant remains, migrates upwards and is collected in a glass jar (Fig. 4f) with a capacity of
0.5-1.1, which is installed on the opening for collecting the mite.

Such a device simulates the structure of the natural mite population in greenhouses. Moving the food along the tiers and laying fresh food in the upper tier repeats the process of spreading the pest from the bottom upwards along the plant. The developed design allowed to increase the yield of the predatory tick nearly three times.

The productivity of the kit per cycle is $2.4 \times 10^{5}$ phytosialus. One breeding cycle lasts 12-14 d. The overall dimensions of the module are $1.3 \mathrm{~m} \times 1.2 \mathrm{~m} \times$ $0.6 \mathrm{~m}$, with weight $50 \mathrm{~kg}$.

\section{Conclusions}

As a result of the studies, sets of technological equipment for the mass breeding of trichogramma, brakon, green lacewing and phytoseuulus were developed. The main technical and economic characteristics of new equipment, such as the number of insects in the cycle that are contained or produced from a unit of the area or volume of the device, provide the necessary profitability of production. 


\section{Acknowledgments}

The author expresses his gratitude to the Biotechnics Engineering and Technology Institute, in which this work was carried out in accordance with the scientific and technical programs of the National Academy of Agrarian Sciences of Ukraine.

\section{References}

[1] Lenteren, J. C. 2012. "The State of Commercial Augmentative Biological Control: Plenty of Natural Enemies, but a Frustrating Lack of Uptake." BioControl 57 (1): 1-20.

[2] Morales-Ramos, J., Rojas, M. G., and Shapiro-Ilan, D. 2014. Mass Production of Beneficial Organisms: Invertebrates and Entomopathogens. London: Elsevier Inc..

[3] Starchevsky, I. 1994. "Prospects for the Development of the Biological Method of Plant Protection." Machineries of Agroindustrial Complex 9-10: 2-4. (in Ukrainian)

[4] Starchevsky, I., Goncharuk, A., and Shaiken, B. 1994. "Industrial Production of Entomo Culture." Machineries of Agroindustrial Complex 9-10: 8-10. (in Ukrainian)

[5] Khodorchuk, V. J., and Bespalov, I. N. 2016. "Techno-Economic Optimization of Modular Complexes of Industrial Production of Entomophages." In Proceedings of International Scientific-Practical Conference "the Biotechnological Systems of Production and Application of Biological Plant Protection Tools in Agriculture", 261-4. (in Ukrainian)

[6] Bespalov, I. M., Sapozhnikova, M. M., and Belousov, Y. V. 2010. "Optimization of the Structure of the Cages for the Breeding of Arthropods." News of Agrarian Sciences 4: 47-9. (in Ukrainian)

[7] Dubrovin, V., Golub, G., and Marus, O. 2012.
"Production of Entomological Preparation of Trichogramma." Motpol. Lublin-Kiev. 14 (3): 9-19. (in Russian)

[8] Leshishak, O. 2014. "Improvement of Trichogramma Mass Rearing Technologies." Quarantine and Plant Protection 7: 14-5. (in Ukrainian)

[9] Rudik, L., Targonya, V., Grogulenko, D., Belchenko, V., and Molchanova, O. "Industrial Biotechnology for the Production of Entomophagous of Habrobracon for Biological Protection of Plants." Machineries and Technologies of Agroindustrial Complex 12 (59): 29-33. (in Ukrainian)

[10] Molchanova, E. D., Motorko, I. S., and Chtrnova, I. S. 2012. "The Main Principles of the Organization of Production of Entomophagous of Habrobracon." News of Agrarian Science of the Southern Region, Interagency Thematic Scientific Collection, Odessa. (in Ukrainian)

[11] Khodorchuk, V., Dubrovin, V. V., Targonya, V., Belousov, Y., Sapozhnikova, M., Klymenko, V., and Belchenko, V. 2012. "Industrial Technology of Production of an Entomophage of Green Lacewing for Biological Protection of Plants." Machineries and Technologies of Agroindustrial Complex 12 (39): 19-21. (in Ukrainian)

[12] Sapozhnikova, M. V., Belousov, Y., and Belchenko, V. 2012. "Technology of Chrysoperla carnea Larvae Feeding Up.” News of Agrarian Science of the Southern Region, Interagency Thematic Scientific Collection, Odessa. (in Ukrainian)

[13] Sapozhnikova, M. V., Belousov, Y., and Belchenko, V. 2012. "Mass Rearing Technology of Chrysoperla affinis Steph. (Neuroptera: Chrysopidae)." News of Agrarian Science of the Southern Region, Interagency Thematic Scientific Collection, Odessa. (in Ukrainian)

[14] Visystak, V., and Limar, I. 2017. "Technology and Methods of Packaging of Predatory Mite Phytoseulatus." Scientific and Information Bulletin of Completed Scientific Researches, Kiev. (in Ukrainian) 